\title{
Comparison between a Novel Liquid Switch and a GaAs MMIC Switch for Reconfiguring the Operating Frequency of a Vivaldi Antenna
}

\author{
Cristina Borda-Fortuny, Kin-Fai Tong, Kevin Chetty, Kai-Kit Wong \\ University College London \\ London, WC1E 7JE \\ Email: cristina.borda.12@ucl.ac.uk
}

\begin{abstract}
This article proposes a novel liquid switch to reconfigure the operating frequency of a frequency-independent antenna. A Vivaldi antenna using a low-cost GaAs MMIC RF switch is used as a landmark to compare the measured results. Two prototypes are measured in an anechoic chamber and the results have been compared. The antennas operate in two modes: low-band mode at $3 \mathrm{GHz}$ with $11 \mathrm{dBi}$ of gain and high-band mode operating at $4.5 \mathrm{GHz}$ with a measured gain of $10.8 \mathrm{dBi}$. The reconfigurable Vivaldi antenna proposed here presents high isolation between operating bands, a minimum of $12 \mathrm{~dB}$, while maintaining high gain and stable radiation pattern which is suitable for cognitive radio applications.
\end{abstract}

\section{INTRODUCTION}

Because of the rapid proliferation of wireless communications systems, the electromagnetic spectrum has become heavily congested. To address this challenge, new wireless communication systems are required to be cognitive and reconfigurable [1]. Currently, antennas are a critical part of the communication systems. Although big limitations appear because of their incapability to be dynamically adapted to changing environments [2], [3].

By selecting the bands of interest in an ultra-wideband antenna, the reconfiguration process of the operating band of an antenna can be facilitated. Narrowband antennas can only operate in a narrow frequency range and impedance matching may be complicated when functioning in a wide frequency range. The frequency-independent Vivaldi antenna is a good candidate for a frequency-reconfigurable antenna. Vivaldi antennas provide high gain and stable beamwidth and consistent direction of the main lobe in their wide operating band [4].

Some work on reconfigurable Vivaldi antennas has been reported in [5]-[7]. Resonating rings are introduced to a Vivaldi antenna to obtain a tuning narrow band or a wide band. Although good isolation between bands is achieved in these designs, the gain is compromised by less than $5 \mathrm{~dB}$.

To provide high isolation and preserve high gain in the Vivaldi antenna an impedance tuning mechanism is proposed in this paper. First, RF switches are considered. In [8] RFMEMS switches are proposed because of their low insertion loss, high isolation and wide operating bandwidth. But they prove to be difficult to integrate in the design as they require a wire bonding machine, the switches are ESD sensible and require an expensive $90 \mathrm{~V}$ driver to operate them. Therefore, a low-insertion loss GaAs MMIC switch is proposed for design A. Another design is proposed to overcome the issues arisen from design A: insertion losses, extra cost the system, the switch biasing lines affect the antenna performance and are not easily integrated in the design. Thus, design B is proposed using a novel liquid switch mechanism. An ionised solution is used as conductive liquid to operate as the RF switch in design A. Ionised solutions have been demonstrated to be feasible for antenna design as reported in [9]-[11].

\section{Antenna Geometry}

The proposed antenna is designed on a single microwave substrate, as shown in Fig. 1. The dielectric substrate used is $0.762 \mathrm{~mm}$ Taconic RF-43. The top layer presents a microstrip feed-line ended with a stub. The stub matches the impedance of $50 \Omega$ at the input port to the optimum impedance in the slot. The stub-to-slot intersection is highlighted in red. One RF switch is introduced in the slot. The position of this switch determines the effective length of the stub for maximum signal coupling at the stub-to-slot intersection. Table I indicates the design parameters and their values.

The switch controls the current path while setting maximum coupling in the stub-to-slot intersection to produce a higher operating band. To operate in the lower band, i.e. switch is OFF, the switch enables the current to flow through the full length of the slot allowing the current to couple to the open end of the Vivaldi antenna. For the high band mode, i.e. switch is $\mathrm{ON}$, the length of the slot is shorter coupling the current only at higher frequencies. Outside the operating frequency band, the current is not coupled providing high band isolation.

\section{A. Design A using a GaAs MMIC RF switch}

Design A is proposed using a low-insertion loss GaAs MMIC switch. Hittite HMC550AE is considered as it provides adequate technical specifications from DC to $6 \mathrm{GHz}$. A table summarising the main specifications of this switch is presented in Table II [12].

Compared to other switching technologies, GaAs MMIC switches have an acceptable insertion loss and bandwidth, one of the fastest switching speeds and low actuation voltage and bias current. Despite their isolation, which is close to a 


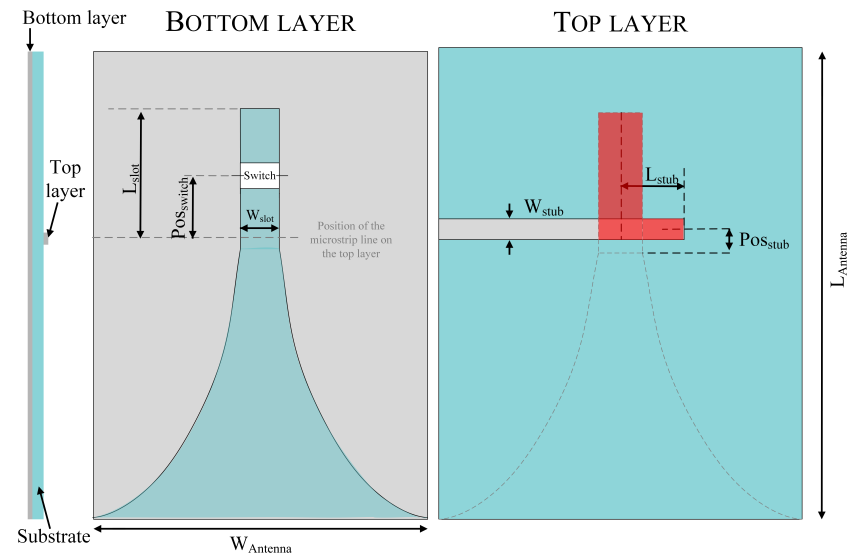

Fig. 1. Proposed geometry for a reconfigurable Vivaldi antenna.

TABLE I

VALUe for the Design Parameters

\begin{tabular}{|c|c|}
\hline Design parameters & Dimensions $(\mathrm{mm})$ \\
\hline $\mathbf{L}_{\text {antenna }}$ & 250.0 \\
$\mathbf{W}_{\text {antenna }}$ & 150.0 \\
$\mathbf{L}_{\text {slot }}$ & 60.0 \\
$\mathbf{W}_{\text {slot }}$ & 1.22 \\
Pos $_{\text {switch }}$ & 40.0 \\
$\mathbf{L}_{\text {stub }}$ & 7.98 \\
$\mathbf{W}_{\text {stub }}$ & 1.5 \\
Pos $_{\text {stub }}$ & 15.8 \\
\hline
\end{tabular}

TABLE II

ChARACTERISTICS OF THE HMC550AE GAAs MMIC SwITCH

\begin{tabular}{|c|c|}
\hline Insertion loss at $\mathbf{1} \mathbf{~ G H z}$ & $0.7 \mathrm{~dB}$ \\
\hline Isolation at $\mathbf{1} \mathbf{~ G H z}$ & $25 \mathrm{~dB}$ \\
\hline Switching speed & $40 \mathrm{~ns}$ \\
\hline Bandwidth & $\mathrm{DC}-6000 \mathrm{MHz}$ \\
\hline Actuation voltage & $1.2-5 \mathrm{~V}$ \\
\hline Bias current & $0.2 \mu \mathrm{A}$ \\
\hline
\end{tabular}

PIN diodes isolation, it is a good candidate for reconfigurable antennas.

A separating cut is required at the end of the slot to separate the two wings of the Vivaldi antenna for the RF switch to operate properly at DC. Fig. 2 presents the fabricated prototype of design A. The prototype is fabricated using an etching technique in the university's workshop. A digital control circuit board is designed to operate the RF switch. The RF switch is manually soldered at the top layer and it connects through vias to the slot at the bottom layer.

The measured S-parameters of the proposed RF switch are embedded in a commercial full-wave EM simulation software (CST Microwave Studio [13]) to obtain results closer to reality.

\section{B. Design B using a novel liquid switch}

A novel liquid switch is considered in design B. The liquid switch is first designed using CST Microwave Studio as shown

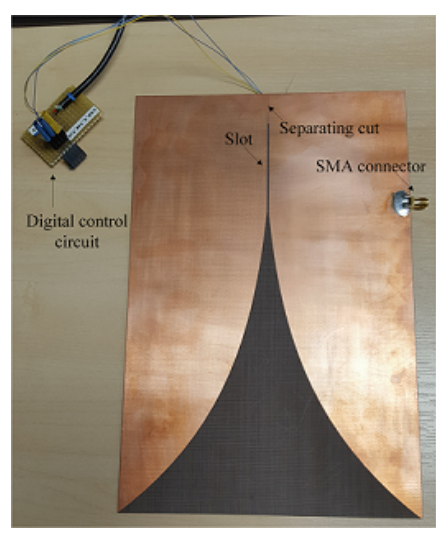

(a) Bottom layer

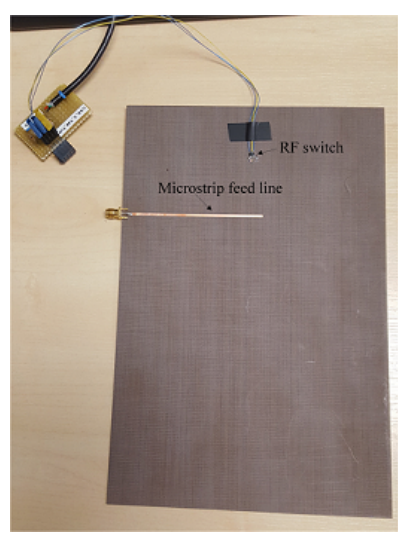

(b) Top layer
Fig. 2. Bottom layer and top layer of the prototype fabricated of design A.

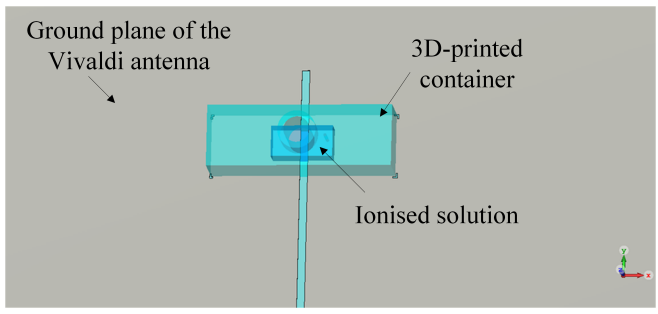

Fig. 3. Liquid switch designed in CST.

in Fig. 3. The CAD design is exported and 3D printed using a Formlabs Form1+ SLA 3D printer. It is attached into the Vivaldi antenna at a specific location to match a higher band. If this location is adjusted the operating frequency at the higher band can be tuned. The ionised solution is $2 \mathrm{~mol} \mathrm{KCl}$ water solution. Its permittivity properties are measured in the lab using Agilent 85070E Dielectric Probe Kit and imported into CST for optimisation.

Currently a syringe is used to introduce the ionised solution into the 3D-printed container. In practical case, a micropump or a diaphragm system can be adopted to automatically tune the operating frequency.

Fig. 4 displays the fabricated prototype for design B. The prototype is measured in the university's anechoic chamber and the results are presented in Section III.

\section{Measured Results}

Fig. 5 compares the measured reflection coefficient and Fig. 6 compares the gain for design A using a GaAs MMIC switch and design $\mathrm{B}$ using a novel liquid switch to control the operating band. HB indicates high band and LB indicates low band. Table III displays the measured results in low-band mode and Table IV presents the measured results in high-band mode.

Looking at the reflection coefficient, there is a frequency shift. Design B is matched $400 \mathrm{MHz}$ lower in frequency than design A. This is because of the effect of the case in the slot and the ionised solution (measured $25 \mathrm{~S} / \mathrm{m}$ at $20^{\circ} \mathrm{C}$ ) not being as conductive as copper $\left(5.96 \cdot 10^{7} \mathrm{~S} / \mathrm{m}\right.$ at $\left.20^{\circ} \mathrm{C}\right)$, 


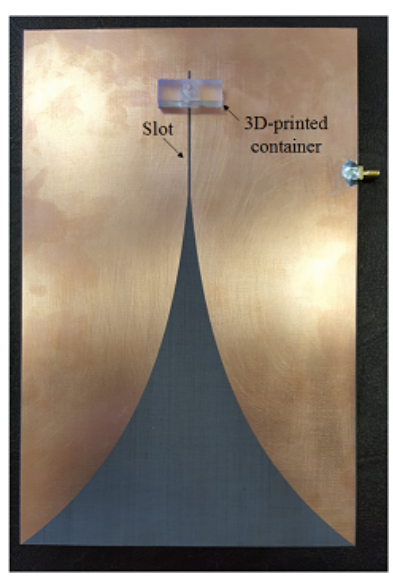

(a) Bottom layer

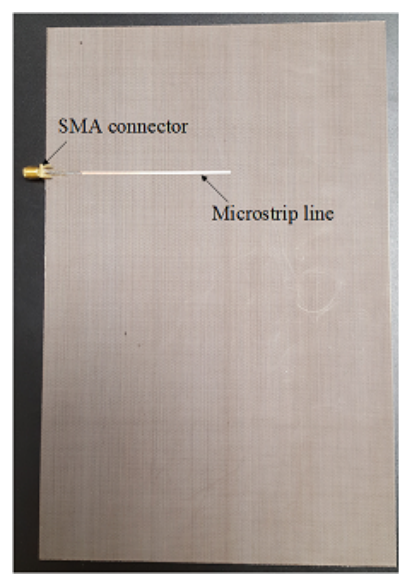

(b) Top layer
Fig. 4. Bottom layer and top layer of the prototype fabricated of design B.

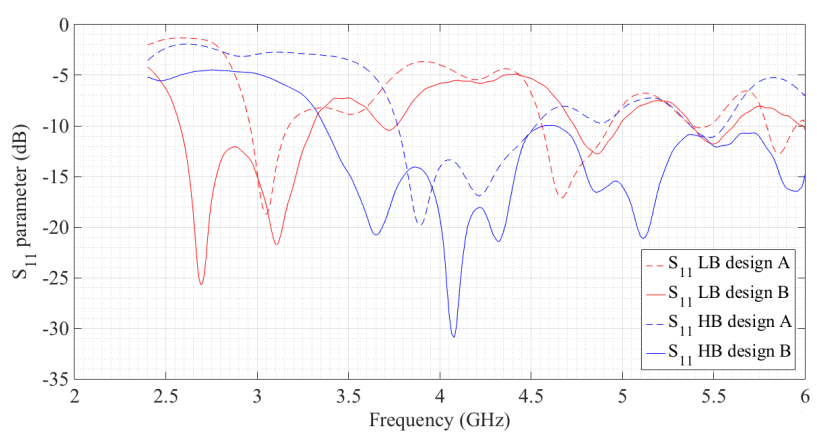

Fig. 5. Measured reflection coefficient compared for design A and design B.

TABLE III

Low-Band Measured Results for Design A and Design B

\begin{tabular}{|c|c|c|}
\hline & Design A & Design B \\
\hline Operating frequency & $3.1 \mathrm{GHz}$ & $3.15 \mathrm{GHz}$ \\
\hline Gain & $10.5 \mathrm{dBi}$ & $11 \mathrm{dBi}$ \\
\hline Isolation & $14 \mathrm{~dB}$ & $12 \mathrm{~dB}$ \\
\hline
\end{tabular}

making the liquid like an absorber and matching it in lower frequencies. However, the gain at these lower $400 \mathrm{MHz}$ is very low compared to the rest of the band. Therefore, this shift is not critical as design B still operates at the frequencies of interest: $3 \mathrm{GHz}$ and $4.5 \mathrm{GHz}$. From $4.6 \mathrm{GHz}$ and up the high band of design $\mathrm{B}$ is again well matched at $5 \mathrm{GHz}$ which design A is not. This can be because of the losses in the liquid material and a poor CST model of the liquid switch which carries the error at higher frequencies. For the gain the main difference is the shift for the rejected frequency in lowband mode. This is because of the liquid case affecting the slot length at the rejected frequencies.

\section{CONCLUSION}

The frequency-independent Vivaldi antenna is proposed as the basis for a frequency reconfigurable antenna design. To tune the operating frequency two designs are proposed. Design

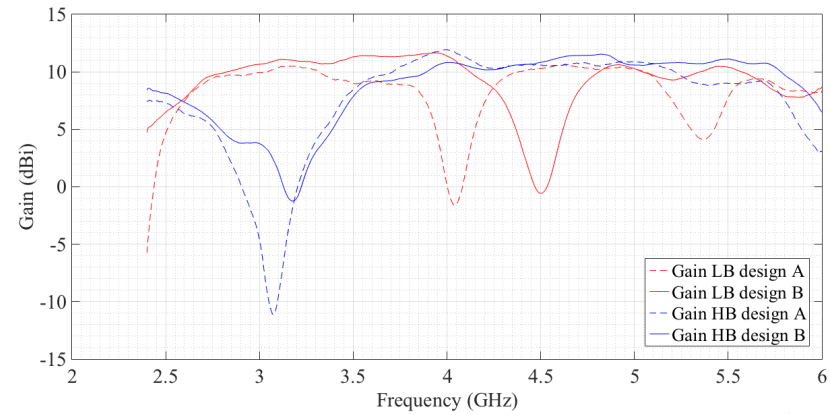

Fig. 6. Measured gain compared for design A and design B.

TABLE IV

High-Band Measured Results for Design A and Design B

\begin{tabular}{|c|c|c|}
\hline & Design A & Design B \\
\hline Operating frequency & $4 \mathrm{GHz}$ & $4.5 \mathrm{GHz}$ \\
\hline Gain & $12 \mathrm{dBi}$ & $11 \mathrm{dBi}$ \\
\hline Isolation & $22 \mathrm{~dB}$ & $12.5 \mathrm{~dB}$ \\
\hline
\end{tabular}

A introduces an RF switch in the slot to match the band of interest. While design B uses a novel liquid switch created to provide higher flexibility and lower impact on the antenna performance due to biasing lines. The prototypes of these two designs are measured in an anechoic chamber and present good agreement.

\section{REFERENCES}

[1] Z. N. Chen, Handbook of Antenna Technologies. Springer, 2015.

[2] J. Bernhard, Reconfigurable Antennas, ser. Synthesis Lectures on Antennas and Propagation Series. Morgan \& Claypool Publishers, 2007.

[3] B. Cetiner, H. Jafarkhani, J.-Y. Qian, H. J. Yoo, A. Grau, and F. De Flaviis, "Multifunctional reconfigurable MEMS integrated antennas for adaptive MIMO systems," IEEE Communications Magazine, vol. 42, no. 12, pp. 62-70, 2004

[4] P. J. Gibson, "The Vivaldi aerial," in 9th European Microwave Conference, 1979., 1979, pp. 101-105.

[5] M. Hamid, P. Gardner, P. Hall, and F. Ghanem, "Multimode vivaldi antenna," Electronics Letters, vol. 46, no. 21, pp. 1424-1425, 2010.

[6] M. Hamid, P. Hall, P. Gardner, and F. Ghanem, "Frequency reconfigurable vivaldi antenna," in Antennas and Propagation (EuCAP), 2010 Proceedings of the Fourth European Conference on, 2010, pp. 1-4.

[7] M. Hamid, P. Gardner, P. Hall, and F. Ghanem, "Switched-band vivaldi antenna," Antennas and Propagation, IEEE Transactions on, vol. 59, no. 5, pp. 1472-1480, 2011.

[8] C. Borda-Fortuny, A. Amiri, and K. F. Tong, "Development of reconfigurable multiple wideband antenna for radar and monitoring applications," in 2015 9th European Conference on Antennas and Propagation (EuCAP), May 2015, pp. 1-5.

[9] C. Murray and R. Franklin, "Independently tunable annular slot antenna resonant frequencies using fluids," IEEE Antennas and Wireless Propagation Letters, vol. 13, pp. 1449-1452, 2014.

[10] M. D. Dickey, "Emerging Applications of Liquid Metals Featuring Surface Oxides," ACS Applied Materials \& Interfaces, vol. 6, no. 21, pp. 18369-18379, 2014.

[11] G. Hayes, J.-H. So, A. Qusba, M. Dickey, and G. Lazzi, "Flexible liquid metal alloy (EGaIn) microstrip patch antenna," IEEE Transactions on Antennas and Propagation, vol. 60, no. 5, pp. 2151-2156, May 2012.

[12] Hittite. (2014, December) HMC550 datasheet. [Online]. Available: http://www.analog.com/media/en/technical-documentation/datasheets/hmc550.pdf

[13] "CST Microwave Studio." [Online]. Available: https://www.cst.com/Products/CSTMWS/ 